\title{
A combined approach for ischemic mitral valve regurgitation: Scar plication and the role of magnetic resonance imaging
}

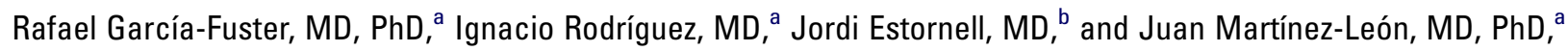 \\ Valencia, Spain
}

$\mathrm{T}$ he incidence and severity of ischemic mitral regurgitation (MR) are higher with posteroinferior infarctions. More localized remodeling without important left ventricular (LV) dilation can result in greater geometric changes with displacement of the posterior papillary muscle (PPM). ${ }^{1}$ A variety of techniques have been advocated to correct

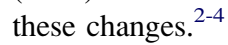

Cardiac magnetic resonance (CMR) has emerged as a new technique that provides detailed information about LV and mitral valve morphology and function with precise myocardial scar assessment. ${ }^{5}$

We report a combined diagnostic-surgical approach for the management of patients with ischemic MR secondary to posteroinferior infarction. It is based on cardiac catheterization, CMR, and perioperative transesophageal echocardiography followed by a 3-staged surgical procedure: selective scar plication, restrictive annuloplasty, and coronary artery bypass grafting.

\section{Clinical Summary}

Between December of 2005 and December of 2006, 4 male patients (median age: 57 years, 51-63 years) underwent this procedure. All had grade 3 to $4+\mathrm{MR}$ at rest and isolated inferior-posterolateral postinfarction scarring detected by CMR (Figure 1, $A$ ), mainly localized in segments 4, 5, 10, and 11 (American Heart Association Consensus about LV walls segments). ${ }^{5}$ Segment 10 (corresponding to PPM) was dyskinetic, and the other 3 segments were akineticdyskinetic. Most viable segments were hypokinetic. Echocardiographic evidence of restrictive leaflet motion (type IIIb) was demonstrated.

Scar plication was performed on the beating heart during cardiopulmonary bypass. The absence of intracavitary thrombus was confirmed by transesophageal echocardiography. The borders of the fibrotic area were identified during ventricular venting, which creates a longitudinal depression corresponding to the scar detected by CMR (Figure 1, B). Stay sutures were passed through the margins, and the center of the infarct was pushed into the LV; $2-0$ polypropylene suture (36-mm needle) was preferred, and deep suture

From the Department of Cardiac Surgery ${ }^{a}$ and Unit of Cardiac Magnetic Resonance, ${ }^{\mathrm{b}}$ University General Hospital of Valencia, Spain.

Received for publication Sept 19, 2007; accepted for publication Dec 18, 2007.

Address for reprints: Rafael García Fuster, MD, PhD, C/Artes Gráficas n ${ }^{\circ}$ 4,esc. izda, pta 3. 46010 Valencia, Spain (E-mail: rgfuster@terra.com).

J Thorac Cardiovasc Surg 2008;135:1169-72

$0022-5223 / \$ 34.00$

Copyright (C) 2008 by The American Association for Thoracic Surgery doi:10.1016/j.jtcvs.2007.12.017 bites were made into both edges of the scar with a continuous mattress suture buttressed with pericardial felt. Plication was limited to the scar and started at the basal portion moving longitudinally toward the apex and close to the adjacent PPM base (Figure 2, A). Both edges were approximated, and the thin infarcted wall was completely invaginated (Figure 2,B).

Patients were revascularized with a mean of $2.4 \pm 1.2$ grafts and restrictive annuloplasty, downsizing by 1 size, was associated. Three 28-mm and one 26-mm Carpentier-Edwards Physio rings were implanted.

\section{Results}

After a mean follow-up of 16 months, MR was absent in 3 patients and trivial in 1 patient. New York Heart Association class decreased from III/IV to II. LV ejection fraction improved from $37 \% \pm 2 \%$ to $55 \% \pm 6 \%$. Preoperative cine CMR does not reveal advanced global LV remodeling (end-diastolic and end-systolic LV volumes: $183 \pm$ $4 \mathrm{~mL}$ and $106 \pm 2 \mathrm{~mL}$ preoperatively vs $171 \pm 3 \mathrm{~mL}$ and $92 \pm 4 \mathrm{~mL}$ postoperatively, respectively) but important local geometric changes with systolic apical displacement of the PPM and marked leaflet tethering (tenting height: $12 \pm 3 \mathrm{~mm}$, tenting area: $1.6 \pm$ $0.5 \mathrm{~cm}^{2}$, PPM distance from anterior mitral annulus: $6.3 \pm 0.3$ $\mathrm{cm})$. Postoperatively, this PPM displacement was corrected, the tenting height was $4 \pm 2 \mathrm{~mm}$, and MR disappeared. See Table 1 for patient data and Figure 3 for CMR imaging views.

\section{Discussion}

Ring annuloplasty is the standard approach for ischemic MR, but recent studies have demonstrated that MR can persist or recur in relation to continued remodeling. Important geometric changes and tethering may be responsible for unsuccessful repair, and some predictors of recurrent MR have been advocated: tenting height greater than $11 \mathrm{~mm}$ or tenting area greater than $1.6 \mathrm{~cm}^{2}$. Annuloplasty alone is not enough in these cases, and adjunctive techniques are needed..$^{2-4}$ We report a 3-staged procedure: selective infarct plication, restrictive annuloplasty, and coronary artery bypass grafting. The objective of plication is restoring local 3-dimensional LV and mitral geometry and eliminating the otherwise deleterious effect of the akineticdyskinetic scarred segments. Since the original report of infarct plication based on 3-dimensional echocardiography findings by Liel-Cohen and colleagues ${ }^{2}$ in a sheep model of ischemic MR, this is one of the first studies presenting infarct plication guided by CMR for ischemic MR in humans. In our patients, despite the important localized LV remodeling and tethering, the results were successful at mid-term follow-up.

Ramadan and colleagues 3 proposed an "everting plication" without annuloplasty, but the avoidance of annuloplasty is not a complete solution because annular dilation is generally present and the infarction may involve the posterior annulus. 

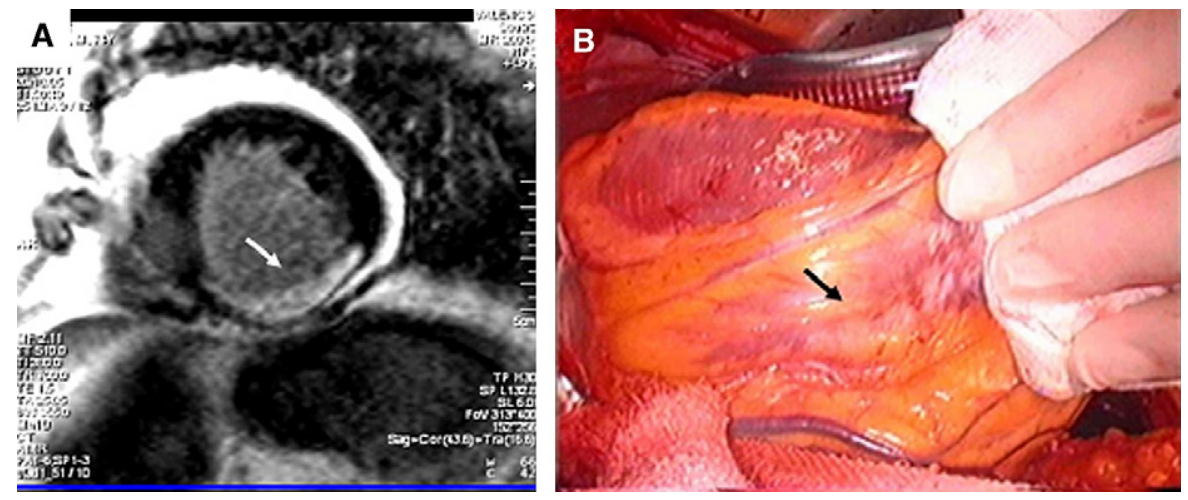

Figure 1. CMR imaging and surgical view. A, Scar at the posterolateral wall of LV. B, The infarct zone was depressed at the posterolateral LV wall during vigorous venting. CMR, Cardiac Magnetic resonance; $L V$, left ventricle.

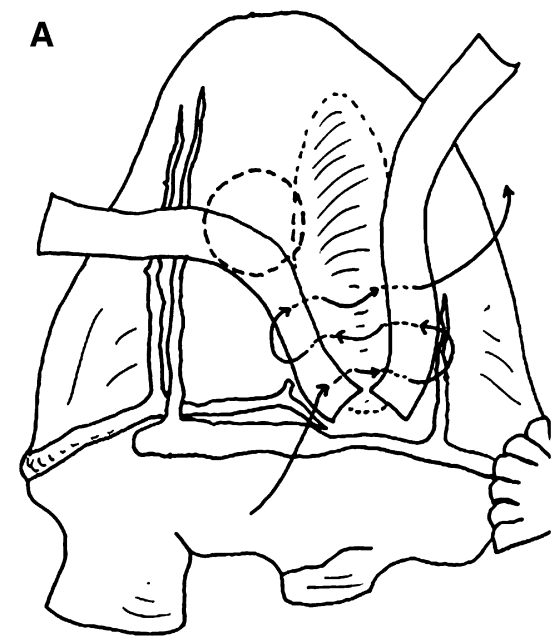

B

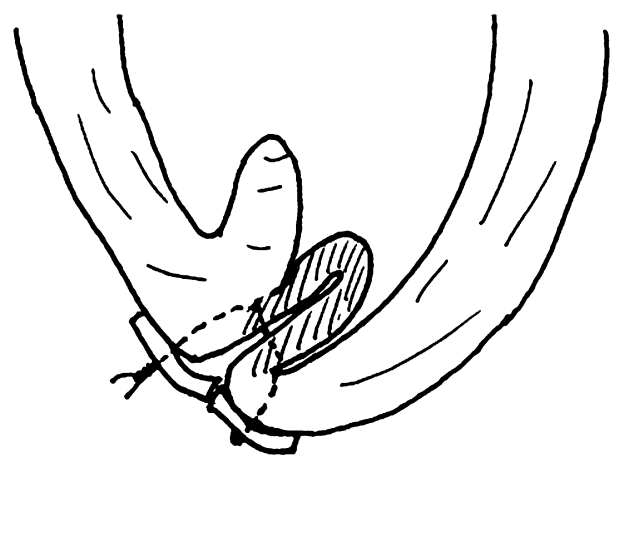

Figure 2. Scar plication. A, Longitudinal plication of the posterolateral scar. B, The thin infarcted wall was completely invaginated.

TABLE 1. Quantitative magnetic resonance data of left ventricular geometry and systolic function

\begin{tabular}{|c|c|c|c|c|c|c|c|c|}
\hline \multirow[b]{3}{*}{ Data } & \multicolumn{2}{|c|}{ Patient 1} & \multicolumn{2}{|c|}{ Patient 2} & \multicolumn{2}{|c|}{ Patient 3} & \multicolumn{2}{|c|}{ Patient 4} \\
\hline & \multicolumn{2}{|c|}{ Male, 60 y } & \multicolumn{2}{|c|}{ Male, 54 y } & \multicolumn{2}{|c|}{ Male, 59 y } & \multicolumn{2}{|c|}{ Male, 56 y } \\
\hline & Preoperative & Postoperative & Preoperative & Postoperative & Preoperative & Postoperative & Preoperative & Postoperative \\
\hline LVEDV (mL) & 187 & 174 & 179 & 168 & 185 & 172 & 182 & 169 \\
\hline LVESV (mL) & 109 & 96 & 104 & 88 & 105 & 94 & 106 & 90 \\
\hline LVEDd (mm) & 65 & 60 & 60 & 55 & 64 & 59 & 63 & 57 \\
\hline LVESd (mm) & 49 & 43 & 45 & 39 & 47 & 41 & 48 & 40 \\
\hline Tenting height (mm) & 15 & 6 & 9 & 2 & 13 & 5 & 10 & 3 \\
\hline Tenting area $\left(\mathrm{cm}^{2}\right)$ & 2.1 & 0.8 & 1.1 & 0.4 & 1.9 & 0.7 & 1.3 & 0.5 \\
\hline $\operatorname{LVEF}(\%)$ & 35 & 49 & 39 & 61 & 36 & 51 & 38 & 58 \\
\hline
\end{tabular}

$\angle V E D V$, Left ventricular end-diastolic volume; $L V E D d$, left ventricular end-diastolic diameter; $L V E F$, left ventricular ejection fraction; $L V E S V$, left ventricular end-systolic volume; LVESd, left ventricular end-systolic diameter.

Direct relocation of the $\mathrm{PPM}^{4}$ tries to recover the normal mitral geometry, but papillary muscles can be infarcted and fragile, and in patients without significant enlargement of the left atrium and ventricle, the approach of the subvalvular area may be difficult.

\section{Conclusions}

Our selective scar plication combined with annuloplasty and coronary artery bypass grafting may be useful in patients with ischemic MR secondary to posteroinferior isolated infarction with localized 


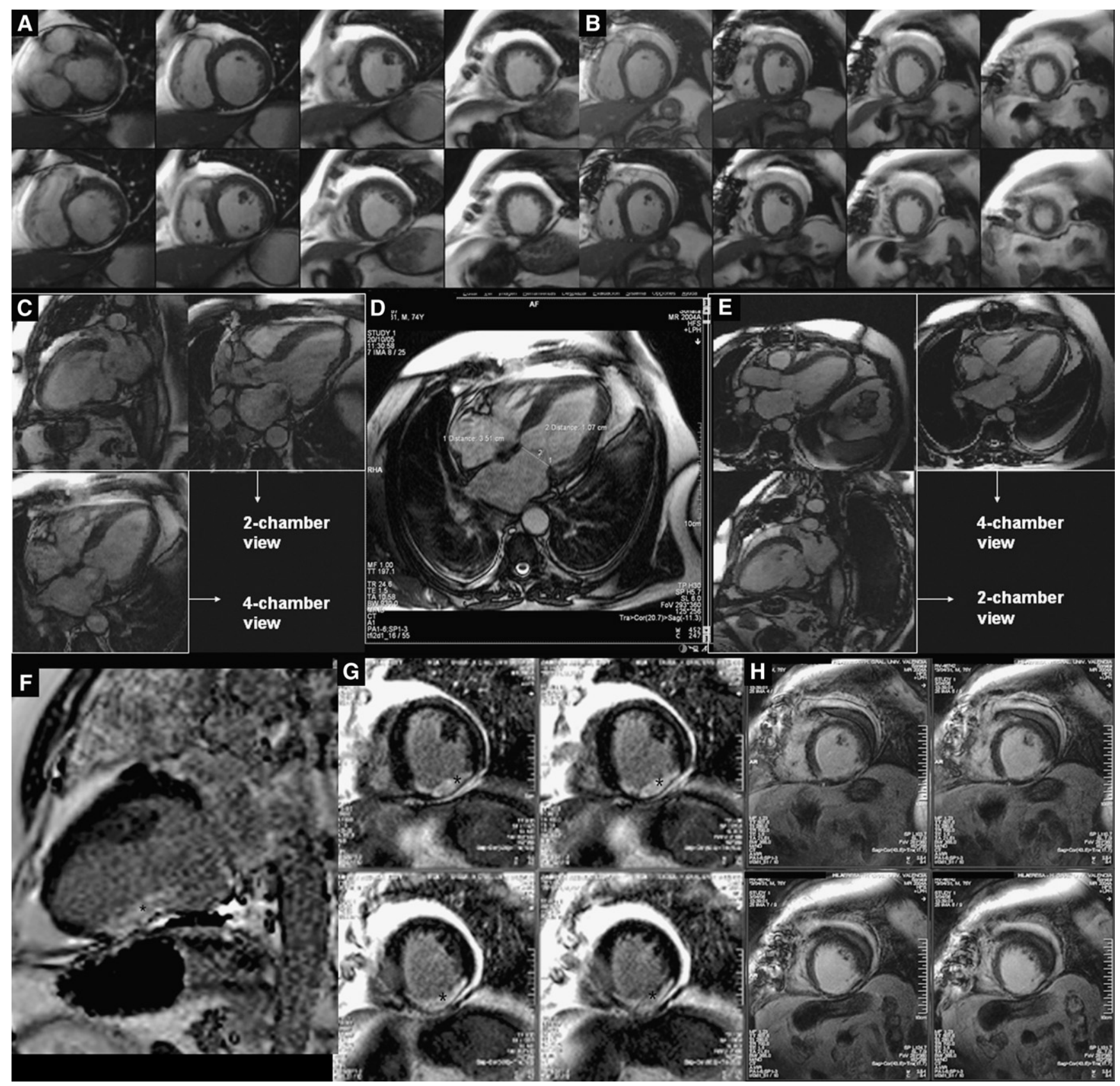

Figure 3. Representative CMR imaging views to assess these patients with ischemic mitral valve regurgitation. $A$ and $B$, Short-axis image, cine magnetic resonance imaging, 2-chamber view from base to apex to measure systolic and diastolic volumes and diameters (A, preoperative; B, postoperative). C, D, and E, Long-axis image, cine magnetic resonance imaging, 4 and 2-chamber views ( $C$ and $D$, preoperative; $E$, postoperative). Tenting height (2) is defined as the shortest distance during systole from the coaptation point of the anterior and posterior mitral leaflets to the mitral annular plane (1). Tenting area is defined as the smallest area during systole bounded by the leaflets and the mitral annular plane. F, G, and $H$, Long-axis $(F)$ and short-axis (G, H), 2-chamber views (F and G, preoperative; $H$, postoperative). Delayed enhancement sequence shows large area of subendocardial necrosis of the midventricular inferior wall. Scarred posteromedial papillary muscle (F). CMR, Cardiac magnetic resonance. 
LV geometric changes. CMR may be an important diagnostic technique to guide the decision-making process. Our initial experience has been encouraging, but future studies with a greater number of patients and long-term follow-up are warranted.

\section{References}

1. Kumanohoso T, Otsuji Y, Yoshifuku S, Matsukida K, Koriyama C, Kisanuki A, et al. Mechanism of higher incidence of ischemic mitral regurgitation in patients with inferior myocardial infarction: quantitative analysis of left ventricular and mitral valve geometry in 103 patients with prior myocardial infarction. J Thorac Cardiovasc Surg. 2003;125: $135-43$.

2. Liel-Cohen N, Guerrero L, Otsuji Y, Handschumacher MD, Rudski LG, Hunziker PR, et al. Design of a new surgical approach for ventricular re- modeling to relieve ischemic mitral regurgitation. Insights from 3-dimensional echocardiography. Circulation. 2000;101:2756-63.

3. Ramadan R, Al-Attar N, Mohammadi S, Ghostine S, Azmoun A, Therasse A, et al. Left ventricular infarct plication restores mitral function in chronic ischemic mitral regurgitation. $J$ Thorac Cardiovasc Surg. 2005;129:440-2.

4. Kron IL, Green GR, Cope JT. Surgical relocation of the posterior papillary muscle in chronic ischemic mitral regurgitation. Ann Thorac Surg. 2002;74:600-1.

5. Bayes de Luna A, Wagner G, Birnbaum Y, Nikus K, Fiol M, Gorgels A, et al. A new terminology for left ventricular walls and location of myocardial infarcts that present $\mathrm{Q}$ wave based on the standard of cardiac magnetic resonance imaging: a statement for healthcare professionals from a committee appointed by the International Society for Holter and Noninvasive Electrocardiography. Circulation. 2006;114: 1755-60.

\title{
Recurrence of myxoma in the left ventricle with concurrent cerebral fusiform aneurysms after previous atrial myxoma surgery
}

\author{
John-Peder Escobar Kvitting, MD, PhD, Jan Engvall, MD, PhD, ${ }^{\mathrm{b}}$ Mats Broqvist, MD, PhD, ${ }^{\mathrm{c}}$ Stefan Franzén, MD, ${ }^{\mathrm{a}}$ \\ Mats Andersson, MD, ${ }^{d}$ Ulf Ohlsson, MD, ${ }^{e}$ and Niels Erik Nielsen, MD, PhD, ${ }^{c}$ Linköping and Oskarshamn, Sweden
}

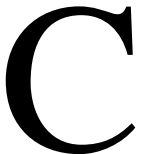
ardiac myxoma belongs to the possible findings in a workup after cerebral stroke, despite being an uncommon disease with an incidence of 0.5 per million population per year. ${ }^{1}$ After surgical removal, cardiac myxoma has a recurrence rate of $2 \%$ to $3 \%$, higher in patients with familial myxomas $(30 \%-75 \%)^{2,3}$ Most new tumors develop in the atria. A local recurrence suggests incomplete resection at the primary operation. Whether recurrent myxoma is a result of dissemination of tumor cells at the first surgical procedure or a multifocal disease remains unclear. $^{4}$ Since the introduction of echocardiography and cardiac magnetic resonance (CMR) imaging, the follow-up of these patients has improved.

\section{Clinical Summary}

In June of 2005, a 55-year-old female patient, previously healthy without medication or allergies, presented with a left-sided hemipa-

From the Departments of Cardiothoracic Surgery, ${ }^{\text {a Clinical Physiology, }}{ }^{\mathrm{b}}$ Cardiology, ${ }^{\mathrm{c}}$ and Neurology ${ }^{\mathrm{d}}$ Linköping University Hospital, Linköping, Sweden; Department of Medicine, Oskarshamns Hospital, ${ }^{\mathrm{e}}$ Oskarshamn, Sweden.

Supported by a grant from the Swedish Heart-Lung Foundation.

Received for publication Nov 27, 2007; accepted for publication Dec 22, 2007.

Address for reprints: John-Peder Escobar Kvitting, MD, PhD, Department of Cardiothoracic Surgery, Linköping University Hospital, SE - 58185 Linköping (E-mail: johkv@imv.liu.se).

J Thorac Cardiovasc Surg 2008;135:1172-3

$0022-5223 / \$ 34.00$

Copyright $\odot 2008$ by The American Association for Thoracic Surgery doi:10.1016/j.jtcvs.2007.12.020 resis at the emergency department of a local hospital. Cerebral computed tomography scan displayed a 1-cm frontotemporal lesion without bleeding. Her symptoms abated in 24 hours. Electrocardiography showed normal sinus rhythm, and the chest x-ray was normal. Transthoracic echocardiography (TTE) revealed a myxoma in the left atrium. The tumor was removed with a biatrial approach under cardioplegic arrest. The postoperative course was uneventful. Histology showed a benign myxoma with an undetermined surgical margin toward the insertion point.

In December of 2005, the patient had a new stroke. Recurrent myxoma was suspected. However, several echocardiograms (transthoracic and transesophageal) and a CMR study in April of 2006 showed normal results (Figure 1,A). Carotid ultrasound showed no plaques. Immunologic studies revealed normal immunoglobulins and positive test results for antinuclear antibodies and rheumatoid factor. Anti-citrullin antibodies, extractable nuclear antigen, and antineutrophil antibodies were negative. Lyme disease was ruled out. Lupus anticoagulant was found, with negative anticardiolipin antibodies but no sign of cutaneous vasculitis. The workup implicated cerebral vasculitis; thus, a high-dose steroid regimen was begun and acetylsalicylic acid was administered. A cerebral 4-vessel angiography was performed that showed small aneurysms (Figure 2). The tentative diagnosis was fusiform cerebral aneurysms after myxoma surgery, and during an outpatient consultation the patient was recommended to undergo an annual TTE and a cerebral biopsy to confirm the diagnosis. On the following day, the patient returned to her local hospital with a new stroke. TTE detected a tumor in the apical part of the left ventricle (LV). On CMR, the tumor measured $23 \times 27 \mathrm{~mm}$ with its base at the midportion of the lateral wall (Figure $1, B$ ). Reevaluation of all imaging studies did not reveal any previous signs of ventricular myxoma. The patient was taken to surgery. Heparin-coated tubing was used in the bypass circuit to reduce the heparin dose and prevent bleeding 